\title{
Dificuldades e pensamento matemático avançado evidenciados por graduandos em tarefas sobre demonstrações matemáticas
}

\author{
Debora Cristiane Barbosa Kirnev \\ Professora, Unopar- Universidade Norte do Paraná \\ deborabarbosa09@yahoo.com.br
}

\author{
Angela Marta Pereira das Dores Savioli \\ Professora, UEL- Universidade Estadual de Londrina \\ angelamarta@uel.br
}

\begin{abstract}
Resumo
O presente artigo é parte de uma investigação que buscou elencar dificuldades relacionadas às formas de demonstrações matemáticas evidenciadas em registros escritos de graduandos do curso de matemática de uma universidade norte paranaense em tarefas abordando conjuntos e funções. A discussão e as análises ocorreram apoiando-se em tipos de demonstrações e em processos de desenvolvimento do pensamento matemático avançado, verificando que os processos de representação foram mais evidenciados que os de abstração. Concluiu-se que as dificuldades evidenciadas nos registros escritos dos participantes da pesquisa foram: a escolha da forma de demonstração, dificuldades inerentes ao conteúdo e à escrita na linguagem matemática ou materna.
\end{abstract}

Palavras-chave: Educação Matemática. Demonstrações. Pensamento Matemático Avançado. Conjuntos. Funções.

\section{Difficulties and advanced mathematical thinking evidenced by undergraduate students in activities of mathematical demonstrations}

\begin{abstract}
This article is part of a research that intends to elucidate difficulties related to the forms mathematical demonstrations evidenced in written records of undergraduate students in Mathematic course from a northern university in Paraná based on tasks addressing sets and functions. The discussion and analysis were based on types of demonstrations and process development of advanced mathematical thinking, noticing that the representation processes were more evident than the abstraction. It was concluded that the difficulties highlighted in the written records of the survey participants were: the choice of demonstration form, difficulties inherent in the content and in writing mathematically or in mother tongue.
\end{abstract}

Keywords: Mathematical Education. Demonstrations. Advanced Mathematical Thinking. Sets. Functions. 


\section{Introdução}

Apresentamos os resultados de uma pesquisa que investigou dificuldades encontradas por graduandos na resolução de tarefas envolvendo demonstrações matemáticas. Realizamos uma discussão a respeito de demonstrações, formas de demonstrações e sua relação com o pensamento matemático avançado ${ }^{1}$. Demonstrações e P.M.A. permeiam todo curso de matemática sendo essenciais para a formação do estudante, desenvolvendo o raciocínio lógico e o poder de argumentação.

De acordo com Moreira e David (2005), em matemática, é necessário a construção axiomática do conteúdo estudado, apoiado em definições e teoremas anteriormente estabelecidos para provar novos resultados, para tanto, definições formais e demonstrações rigorosas são elementos importantes para a sistematização teórica.

Rav (1999), Hanna e Barbeau (2008) atestam que as demonstrações são o cerne da matemática e que existem dificuldades no desenvolvimento do raciocínio matemático de estudantes de graduação, ao considerar que as demonstrações são essenciais para o desenvolvimento da matemática.

Em um segundo momento, apresentamos como ocorreu a aplicação de tarefas abordando conjuntos e funções a graduandos de um curso de matemática e como se deu a análise dos registros escritos desses estudantes, apoiados em Balacheff (1987) e Dreyfus (1991), na busca de elementos do P.M.A. e das dificuldades apresentadas pelos estudantes em relação à demonstração e às formas de demonstrações. Interessou-nos as escolhas feitas pelos participantes quando eram apresentados às tarefas, bem como o modo como procederam às demonstrações. Concordamos com Ponte et al, (1997) quando apontam como se desenvolve o raciocínio matemático diante de uma questão a resolver. Esse raciocínio matemático determina que o graduando compare várias situações de aprendizagem, a fim de estabelecer relações entre essas situações, e exige que desenvolva competências e habilidades em demonstrações.

\section{Referencial Teórico}

Uma demonstração seria “[...] uma sucessão articulada de raciocínios lógicos que permite mostrar que um resultado proposto é consequência de princípios previamente fixados e de proposições já estabelecidas" (DOMINGUES E IEZZI, 2003, p.17). Quando nos referimos a demonstrações matemáticas, a argumentação, a qual é utilizada para provar se uma proposição é verdadeira ou falsa, torna-se imprescindível, bem como o raciocínio lógico dedutivo. Concordamos que

\footnotetext{
${ }^{1}$ Utilizaremos P.M.A. para indicar pensamento matemático avançado.
} 
argumentar e raciocinar de maneira dedutiva não são tarefas simples e, portanto, apresentam dificuldades para estudantes nas primeiras séries de um curso de matemática.

Há necessidade da racionalidade para uma prova dedutiva, como afirma Balacheff (2004), pois promove a construção do raciocínio lógico. O autor esclarece que racionalidade:

[..] nos permite à razão e decidir [...] é a base de qualquer processo provado. [...] em geral e sua relação com a matemática em particular, é um ponto-chave para nossa compreensão de qualquer trabalho no nosso campo de pesquisa. Um pode aceitar, não com muita dificuldade, que prova depende do conteúdo e contexto.

(BALACHEFF, 2004, p. 2)

Balacheff (1987) categoriza dois tipos de demonstrações denominadas de prova pragmática e prova conceitual. As provas pragmáticas são produzidas por sujeitos baseados em fatos e ações, contudo não contemplando um formalismo lógico; podem ser denominadas "mostrações", pois os resultados são, em sua maioria, por meio de exemplos. As provas conceituais se caracterizam por formulações de propriedades e as prováveis relações entre elas. Balacheff (1987) afirma haver diversos níveis de provas pragmáticas e provas conceituais caracterizados no quadro um.

Quadro 1- caracterização dos níveis de provas

\begin{tabular}{|c|c|l|}
\hline Tipos & \multicolumn{1}{|c|}{ Nívei } & \multicolumn{1}{c|}{ Caracterização } \\
\hline \multirow{2}{*}{ Provas pragmáticas } & $\begin{array}{c}\text { Empirismo } \\
\text { ingênuo: }\end{array}$ & $\begin{array}{l}\text { lonsiste em afirmar a verdade de uma proposição após a } \\
\text { verificação de alguns casos. É considerado o primeiro } \\
\text { passo no processo de generalização. }\end{array}$ \\
\cline { 2 - 4 } & $\begin{array}{c}\text { Experimento } \\
\text { crucial: }\end{array}$ & $\begin{array}{l}\text { consiste em afirmar a verdade de uma proposição após a } \\
\text { verificação para um caso especial, geralmente não } \\
\text { familiar. }\end{array}$ \\
\hline $\begin{array}{c}\text { Transição entre } \\
\text { provas pragmáticas } \\
\text { e provas conceituais }\end{array}$ & $\begin{array}{c}\text { Exemplo } \\
\text { genérico: }\end{array}$ & $\begin{array}{l}\text { consiste em afirmar a verdade de uma proposição após a } \\
\text { manipulação de alguns exemplos de modo a deixá-los } \\
\text { com uma característica que representa uma classe de } \\
\text { objetos. }\end{array}$ \\
\hline Provas conceituais & $\begin{array}{c}\text { Experimento } \\
\text { de } \\
\text { pensamento: }\end{array}$ & $\begin{array}{l}\text { consiste em afirmar a verdade de uma proposição de } \\
\text { forma genérica, porém baseada no estudo de alguns casos } \\
\text { específicos. }\end{array}$ \\
\hline
\end{tabular}

Fonte: Adaptação de Balacheff (1987)

Ressaltamos que o processo em que uma demonstração está envolvida, segundo Mariotti e Balacheff (2008), propicia ao estudante importantes elementos matemáticos, como estratégias e procedimentos, na resolução de problemas. Ao demonstrar uma proposição é necessário ter-se uma ideia do objeto matemático, formar relações com axiomas ou proposições já demonstradas e, no caso de ser um elemento desconhecido, investigar a respeito do mesmo. 
Assim, podemos inferir que demonstrações matemáticas exigem dos graduandos processos mentais $^{2}$ decorrentes do P.M.A.. Para a realização desses processos há a necessidade de abstrair e representar objetos matemáticos e utilizar a argumentação explícita, a forma de raciocínio lógico dedutivo envolvida.

No desenvolvimento de uma demonstração matemática, o resultado apresentado é uma representação simbólica de um processo mental. Tall (1999, p.15) afirma que "alunos iniciantes têm dificuldade ainda maior com a prova antes de atingir familiaridade com o funcionamento da cultura matemática". Familiaridade construída a partir de relações internas do indivíduo, isto é, por meio do pensamento.

Tall (1991, 1995) e Dreyfus (1991) abordam sobre processos mentais decorrentes da prova matemática. Recorremos a esses autores para esclarecermos o termo P.M.A..

Para Tall (1995, p.3), “o pensamento matemático avançado envolve o uso de estruturas cognitivas produzidas por uma ampla gama de atividades matemáticas para a construção de novas ideias que desenvolvam e ampliem um sistema cada vez maior de teoremas estabelecidos". O mesmo autor ainda afirma que o crescimento cognitivo ocorre por meio da "percepção" e "ação" sobre objetos, utilizando manipulação simbólica baseada em definições e teoremas provados.

Dreyfus (1991) afirma que o P.M.A. ocorre por meio de processos mentais ${ }^{3}$ de objetos matemáticos, e o P.M.A. difere do pensamento elementar por proporcionar reflexões sobre a própria experiência matemática ao lidar com problemas não triviais, variedade de processos mentais que se interagem, e complexidade em processos mentais.

Uma particularidade desse pensamento, para Dreyfus (1991), é a complexidade e como são desenvolvidos os processos mentais. Os processos de abstração e representação permitem passar de um tipo de detalhe para outro, e, deste modo, gerenciar a complexidade.

Dreyfus (1991) afirma que estudar o P.M.A. é uma maneira de conseguir conhecimentos teóricos a respeito do que acontece na mente do estudante no processo de aprendizagem devido às representações mentais que são desenvolvidas.

Ao falarmos ou pensarmos a respeito de um objeto matemático, temos uma representação mental deste, que segundo Dreyfus (1991), refere-se a esquemas internos ou de quadro de referências que a pessoa utiliza para interagir com o mundo externo. Nesse sentido, o P.M.A. consistiria em uma variedade de processos mentais interagindo.

\footnotetext{
2 Processos mentais

${ }^{3}$ Representações mentais
} 
Vários processos mentais podem ser elaborados pelos estudantes segundo Dreyfus (1991). Como exemplo, citamos: representação, visualização, generalização, classificação, conjecturar, induzir, analisar, sintetizar, abstrair ou formalizar.

Segundo Dreyfus (1991, p. 32) podemos ter representações mentais ricas ou pobres, depende da flexibilidade entre as representações mentais que sujeito possuí na resolução de problemas. Se o aluno possui elementos insuficientes para articular as representações, “[...] a menor mudança na estrutura de um problema, ou até mesmo em sua formulação, pode bloqueá-los completamente."

Dreyfus (1991) afirma que várias representações mentais de um conceito podem integrarse em uma única representação desse conceito ou complementar outro, assinalando um processo de abstração, que, por promover uma reflexão consciente sobre conceitos a partir de situações matemáticas, é importante para o desenvolvimento do P.M.A.. Além disso, ter várias representações de um conceito auxilia a resolução de problemas.

Para ocorrer o processo de abstração, dois outros processos são necessários, o de generalizar e o de sintetizar. De acordo com Dreyfus (1991), generalizar é derivar ou induzir a partir de indicações, a fim de identificar pontos em comum e, deste modo, expandir domínios de validade. Já sintetizar implica em combinar ou compor partes de tal forma a constituir um todo.

Assim, temos que a abstração contém

[...] o potencial para generalização e sintetização; e vice-versa, torna-se sua finalidade, principalmente a partir desse potencial de generalização e de síntese. A natureza do processo mental de abstração é, contudo, muito diferente da generalização e do de síntese. Abstrair é antes de tudo um processo de construção - a construção de estruturas mentais a partir de estruturas matemáticas, ou seja, de propriedades e relações entre objetos matemáticos. Este processo é dependente do isolamento de propriedades adequadas e estabelecimento de relações. Requer a capacidade de deslocar a atenção dos objetos em si a estrutura das suas propriedades e relações. (DREYFUS, 1991, p. 37)

Com o intuito de sintetizar os processos mentais envolvidos na representação e na abstração, elaboramos, a partir das observações de Dreyfus (1991), o quadro dois com as respectivas caracterizações, o qual usaremos em nossas análises. 
Quadro 2- caracterização de processos mentais envolvidos no P. M. A.

\begin{tabular}{|c|c|}
\hline \multicolumn{2}{|c|}{ PROCESSOS MENTAIS ENVOLVIDOS NA REPRESENTAÇÃO } \\
\hline Visualizar & Converter algo em representação mental. \\
\hline Reconhecer & Relacionar algo que temos em mente com o que visualizamos. \\
\hline Intuir & Perceber relações existentes em diferentes representações. \\
\hline Investigar & Averiguar o desenvolvimento de um processo. \\
\hline Traduzir & Transitar de uma representação de um objeto para outra. \\
\hline Descobrir & Conhecer algo por meio de conexões de representações mentais. \\
\hline Conjecturar & Deduzir a partir da verificação, tradução, descoberta. \\
\hline Classificar & Ordenar e agrupar processos mentais concorrentes. \\
\hline Analisar & Avaliar, criticar, refletir sobre um processo mental. \\
\hline Induzir & Concluir a partir de indícios, verificações. \\
\hline Reconhecer símbolos & Traduzir signos para representações mentais. \\
\hline Manipular símbolos & Associar e traduzir diferentes representações. \\
\hline Definir & Expor claramente o significado de uma representação. \\
\hline Flexibilizar & Relacionar diferentes representações mentais. \\
\hline Compreender & Entender a interação de uma variedade de processos mentais simultâneos. \\
\hline Modelar & Encontrar uma representação matemática para um objeto não-matemático. \\
\hline \multicolumn{2}{|r|}{ 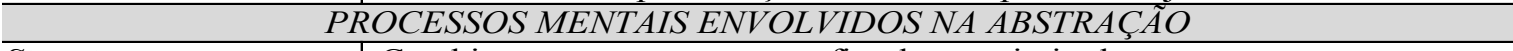 } \\
\hline Sintetizar & Combinar ou compor partes a fim de constituir algo. \\
\hline Formalizar & Criar procedimentos padronizados. \\
\hline Generalizar & $\begin{array}{l}\text { Derivar ou induzir a partir de indícios, para indicar pontos em comum e } \\
\text { ampliar domínios de validade. }\end{array}$ \\
\hline Provar & Argumentar por meio de uma sequência lógica dedutiva. \\
\hline
\end{tabular}

Fonte: Adaptação do Dreyfus (1991)

\section{Aspectos metodológicos}

Como o objetivo da investigação relatada neste artigo era analisar tarefas sobre demonstrações matemáticas a fim de identificar dificuldades apresentadas, optamos, na escolha dos participantes, por graduandos de um curso de Licenciatura em Matemática em uma universidade norte paranaense, matriculados na disciplina de Estruturas Algébricas, na qual são trabalhadas as diferentes formas de demonstrações.

Para cada sujeito integrante da pesquisa, atribuímos uma identificação de $A_{1}$ até $A_{13}$, utilizados para indicar o participante em ambas às aplicações de tarefas.

A primeira proposta de tarefas foi aplicada a treze participantes. Em uma análise inicial dos registros escritos dos estudantes, notamos que apenas duas formas de demonstrações foram contempladas, sendo a direta e por redução ao absurdo. Assim, optamos por uma segunda proposta de tarefas, ocorrida três meses depois da primeira, com questões focando afirmações envolvendo conjuntos e funções. Essa proposta contou com dez estudantes e possibilitou a utilização das formas de demonstrações: direta, contrapositiva e por contraexemplo. Além disso, continha uma questão aberta que permitia mais de uma forma de demonstração. Somente sete dos dez estudantes haviam participado da primeira aplicação.

As tarefas foram desenvolvidas individualmente nas duas aplicações das propostas e, na segunda aplicação, indicamos verbalmente e por escrito, que os estudantes justificassem suas 
escolhas, principalmente se não fizessem a demonstração. Dúvidas referentes à interpretação da proposta não foram esclarecidas.

\section{Análises}

Analisamos os registros escritos por questão para evidenciarmos que dificuldades foram apresentadas. Em cada questão, agrupamos as resoluções similares e analisamos esses agrupamentos, não nos atendo somente aos registros escritos dos sete estudantes que participaram de ambas as aplicações. Escolhemos esta forma para contemplar uma diversidade de registros que enriqueceriam a análise de dificuldades.

Entendemos como dificuldade em demonstrar uma proposição matemática se o estudante: não utilizou uma sequência de argumentos válidos, cujo último argumento seja a conclusão da tese a ser provada; se utilizou uma sequência de argumentos que não atende alguma forma de demonstração: direta, indireta, redução ao absurdo, ou ainda por contraexemplo.

Utilizamos o quadro a seguir para agrupar os tipos de respostas obtidas pelos sujeitos participantes da pesquisa nas resoluções das tarefas.

\section{Quadro 3 - Tipos de respostas}

\begin{tabular}{|l|c|l|}
\hline Coerente & $C_{1}$ & $\begin{array}{l}\text { Consideramos a demonstração correta, ou seja, apresenta uma sequência } \\
\text { lógica dedutiva válida. }\end{array}$ \\
\cline { 2 - 3 } & $C_{2}$ & $\begin{array}{l}\text { Neste caso a demonstração pode conter erros de conteúdo que não } \\
\text { comprometa a forma de demonstração. }\end{array}$ \\
\cline { 2 - 3 } & $C_{3}$ & $\begin{array}{l}\text { Neste caso demonstração não apresenta erros de conteúdo, porém está } \\
\text { incompleta ou a forma de demonstração está inadequada. }\end{array}$ \\
\hline Incoerente & Há tentativa de resolução, porém está incorreta. \\
\hline Em branco & $\begin{array}{l}\text { Não apresenta registro algum na questão. } \\
\text { Justificada }\end{array}$ \\
Fonte: Autorón tentativa de resolução na questão, mas há justificativa de não \\
responder a questão.
\end{tabular}

Após analisarmos e agruparmos os tipos de resoluções surgiu a necessidade de categorizar as dificuldades detectadas, deste modo utilizamos o quadro a seguir para finalizar as análises. 
Quadro 4 - categorização de dificuldades

\begin{tabular}{|c|c|c|c|}
\hline \multirow{3}{*}{$\begin{array}{l}\text { Dificuldade } \\
\text { Desenvolvimento } \\
\text { da forma de } \\
\text { demonstração }\end{array}$} & & & \\
\hline & \multirow[t]{2}{*}{ Argumentação } & Inválida & $\begin{array}{l}\text { Não utiliza uma sequência lógica } \\
\text { dedutiva, é utilizado um argumento falso } \\
\text { que compromete a demonstração. }\end{array}$ \\
\hline & & Incompleta & $\begin{array}{l}\text { utiliza uma sequência lógica dedutiva, } \\
\text { mas, não concluí a demonstração. }\end{array}$ \\
\hline & \multirow[t]{2}{*}{$\begin{array}{l}\text { Estrutura da } \\
\text { demonstração }\end{array}$} & $\begin{array}{l}\text { Distinção entre } \\
\text { hipótese e tese }\end{array}$ & $\begin{array}{l}\text { inicia a demonstração afirmando a tese e } \\
\text { concluí a hipótese. }\end{array}$ \\
\hline & & $\begin{array}{l}\text { Distinção entre } \\
\text { definição e } \\
\text { demonstração }\end{array}$ & $\begin{array}{l}\text { afirma que uma proposição que necessita } \\
\text { ser demonstrada é verdadeira por } \\
\text { definição. }\end{array}$ \\
\hline & \multirow[t]{2}{*}{$\begin{array}{l}\text { Forma de } \\
\text { demonstração }\end{array}$} & $\begin{array}{l}\text { Utiliza } \\
\text { exemplos }\end{array}$ & $\begin{array}{l}\text { a representação simbólica utilizada não se } \\
\text { adéqua a nenhuma forma de } \\
\text { demonstração e sim a exemplos } \\
\text { particulares sobre a proposição. }\end{array}$ \\
\hline & & $\begin{array}{l}\text { Forma } \\
\text { inadequada }\end{array}$ & $\begin{array}{l}\text { inicia-se o processo de prova, porém a } \\
\text { conclusão não é válida por não utilizar a } \\
\text { forma correta, quanto a estrutura direta, } \\
\text { contrapositiva, redução ao absurdo, ou, } \\
\text { contra exemplo. }\end{array}$ \\
\hline \multirow[t]{3}{*}{$\begin{array}{l}\text { Conhecimento do } \\
\text { conteúdo }\end{array}$} & \multicolumn{2}{|l|}{ Conectivos } & $\begin{array}{l}\text { inverte a utilização de conectivos, por } \\
\text { exemplo, e por ou. }\end{array}$ \\
\hline & \multicolumn{2}{|c|}{ Condicional e bicondicional } & $\begin{array}{l}\text { não distingue condição necessária e } \\
\text { condição suficiente. }\end{array}$ \\
\hline & \multicolumn{2}{|c|}{$\begin{array}{l}\text { União, Interseção, } \\
\text { complementar }\end{array}$} & $\begin{array}{l}\text { não argumenta de acordo com as } \\
\text { premissas ou definição. }\end{array}$ \\
\hline \multirow[t]{3}{*}{ Escrita } & \multirow[t]{2}{*}{$\begin{array}{l}\text { Linguagem } \\
\text { materna }\end{array}$} & Clareza & $\begin{array}{l}\text { a representação simbólica utilizada na } \\
\text { escrita é confusa. }\end{array}$ \\
\hline & & Coerência & existe contradição na escrita. \\
\hline & \multicolumn{2}{|c|}{ Linguagem matemática } & $\begin{array}{l}\text { não escreve adequadamente na linguagem } \\
\text { matemática, por exemplo, utiliza } \\
\text { símbolos inadequados, ou, atribui } \\
\text { significado divergente. }\end{array}$ \\
\hline
\end{tabular}

Neste artigo, apresentaremos como exemplo as análises da questão um, item b, e da questão quatro, ambas da segunda proposta de tarefas, baseamo-nos principalmente em Balachef (1987) e em Dreyfus (1991) que afirma que o P.M.A. requer gerenciar a complexidade em uma situação matemática a se resolver. Essas questões escolhidas requerem uma argumentação densa em comparação com as demais, além de que os agrupamentos analisados permitem relacionar essas questões.

\section{Questão um, item b}

1) Seja f: $\mathrm{X} \rightarrow \mathrm{Y}$ uma aplicação e $A \subseteq X$ e $B \subseteq X$. Considerando que as demonstrações matemáticas podem ser realizadas valendo-se das formas direta, contrapositiva ou redução ao absurdo, demonstre, se for possível, as seguintes proposições:

a) $\quad \mathrm{f}(\mathrm{A} \cap \mathrm{B})=\mathrm{f}(\mathrm{A}) \cap \mathrm{f}(\mathrm{B})$

b) $\quad f(A \cup B)=f(A) \cup f(B)$ 
Analisando os tipos de resolução e a coerência das respostas obtidas nesta questão, obtivemos o quadro cinco.

\begin{tabular}{|c|c|c|c|c|c|c|c|c|c|c|c|}
\hline \multirow{2}{*}{\multicolumn{2}{|c|}{ Agrupamentos }} & & & & & & & & & & \\
\hline & & $\overline{4}$ & $\frac{1}{4}$ & $e^{\pi}$ & 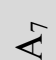 & $\sum^{\infty}$ & $\stackrel{0}{<}$ & $\frac{m}{4}$ & $\stackrel{ \pm}{<}$ & $\stackrel{n}{4}$ & $\stackrel{0}{\pi}$ \\
\hline \multirow[t]{3}{*}{ Coerente } & $C_{1}$ & $\mathrm{X}$ & $\mathrm{X}$ & & & X & & $\mathrm{X}$ & & $\mathrm{X}$ & \\
\hline & $C_{2}$ & & & & & & & & & & \\
\hline & $C_{3}$ & & & $\mathrm{X}$ & $\mathrm{X}$ & & $\mathrm{X}$ & & $\mathrm{X}$ & & $\mathrm{X}$ \\
\hline \multicolumn{2}{|l|}{ Incoerente } & & & & & & & & & & \\
\hline \multicolumn{2}{|l|}{ Em branco } & & & & & & & & & & \\
\hline Justificadc & & & & & & & & & & & \\
\hline
\end{tabular}

Fonte: Autoria própria

Pelo quadro cinco, tivemos as demonstrações dos estudantes agrupadas em $\mathrm{C}_{1}$ ou em $\mathrm{C}_{3}$. Em $\mathrm{C}_{1}$, todos os estudantes provaram as duas inclusões pela forma direta, argumentaram por meio de uma sequência lógica dedutiva e provaram a proposição. Para Balacheff (1987), tivemos casos de experimento de pensamento, ou seja, ocorreram provas conceituais. Vejamos o registro escrito a seguir;

Figura 1 - Registro escrito de $A_{13}$

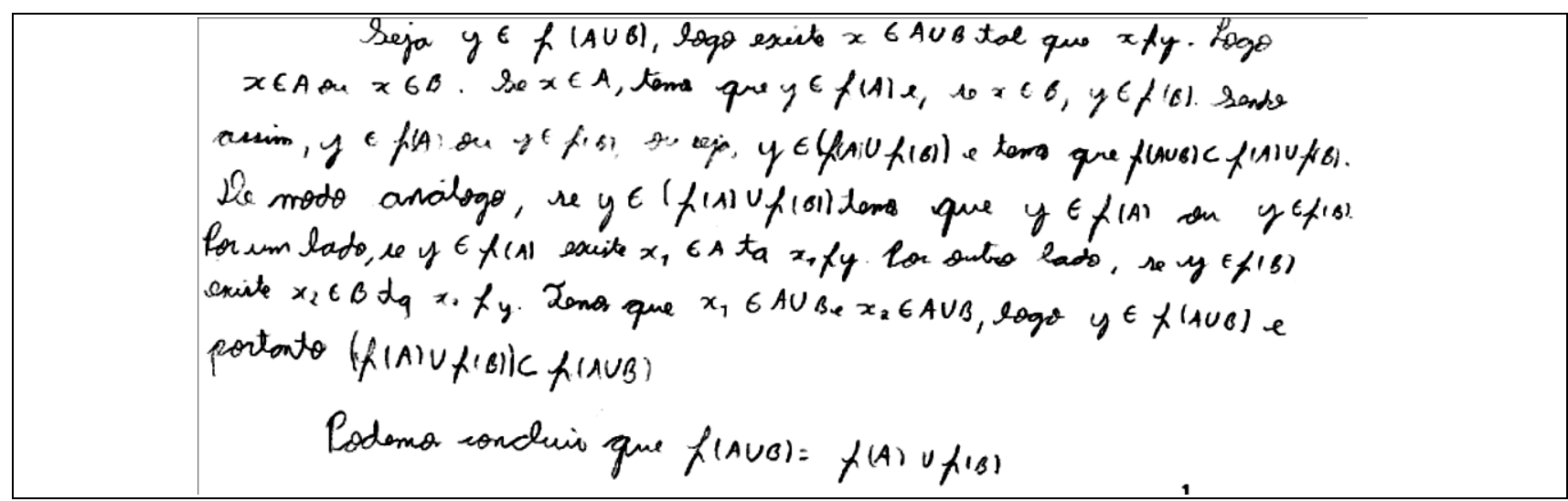

Fonte: registro escrito de $A_{13}$ : questão 1b) da $P_{2}$

Os processos mentais que apareceram em $\mathrm{C}_{1}$, segundo Dreyfus (1991) e pelo quadro dois foram: visualizar, reconhecer, traduzir, classificar, analisar, reconhecer símbolos, manipular símbolos, definir, flexibilizar, compreender, sintetizar, formalizar e provar.

Em relação a $C_{3}$, temos os casos de $A_{4}$ e $A_{7}$ que demonstraram de modo incompleto, de forma algorítmica, sem refletir na argumentação e nos conceitos utilizados, ou seja, não provaram as duas inclusões. Observemos a seguir o protocolo de $\mathrm{A}_{4}$ 
Figura 2 - Registro escrito de $\mathbf{A}_{4}$

\begin{tabular}{|c|c|}
\hline & 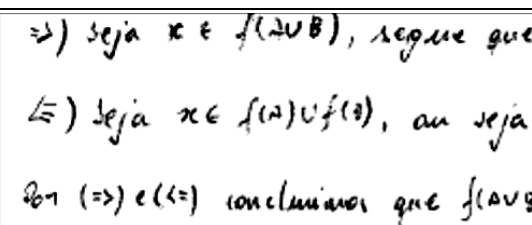 \\
\hline
\end{tabular}

Fonte: registro escrito de $A_{4}$ : questão 1b) da $P_{2}$

Esse tipo de prova Balacheff (1987) classifica como experimento crucial, pois realizaram apenas algumas verificações para afirmar a validade da proposição. Mariotti e Balacheff (2008) asseguram que para demonstrar uma proposição é necessário ter uma noção sobre o objeto matemático, estabelecer relações com axiomas ou afirmações anteriormente provadas e, caso seja um objeto desconhecido, promover a investigação sobre o mesmo. Nos registros escritos desse agrupamento notamos que os estudantes possuem noções sobre o conteúdo e conhecem as sentenças a serem demonstradas, contudo não estabelecem conexões com as definições e axiomas sobre o conteúdo.

Observamos que nessa questão tivemos duas proposições similares, uma falsa e outra verdadeira. Ao elaborar essa questão tínhamos a intenção de investigar, além das dificuldades nas formas de demonstrações e no conteúdo, o processo mental de abstração dos sujeitos da pesquisa, pois, se demonstrassem de forma algorítmica, não reconheceriam que a proposição do item a) necessitava de um contraexemplo.

Observemos no item a seguir um caso de empirismo ingênuo, que segundo Balacheff (1997) pode ser um exemplo de mostração:

Figura 3 - Registro escrito de $A_{14}$

\begin{aligned} \hline Demonstrasas: Sajam $A=\{1,2,3,4\}$ e $B=\{2,3,4,5,6\} \\$. Eutar, se $f(A \cup B)=f^{(A)} \cup f(B) \\$. Temos, $f(A \cup B)=\{1,2,3,4,5,6\}\end{aligned}$

Fonte: registro escrito de $A_{14}$ : questão um b) da $P_{2}$

Para Dreyfus (1991), estes estudantes não construíram o conceito matemático envolvido na demonstração, e, portanto, não desenvolveram os processos mentais de conjecturar ideias, sistematizar, sintetizar e abstrair os conceitos matemáticos.

Notamos que no registro a seguir o sujeito não relacionou a notação com a aplicação de conjuntos, de modo que, identificamos dificuldades com símbolos matemáticos e no uso de equivalências lógicas no registro: 
Figura 4- Registro escrito de $\mathbf{A}_{10}$

Seja $y \in(A \cup B) \Leftrightarrow[(y \in A) \vee(y \in B)] \Leftrightarrow(y \in A) \cup(y \in B) \Leftrightarrow$
$\Leftrightarrow f(A) \cup f(B)$

Fonte: registro escrito de $A_{10}$ : questão um b) da $P_{2}$

O quadro seis sintetiza as dificuldades detectadas nas demonstrações dessa proposição.

\begin{tabular}{|c|c|c|c|c|c|c|c|c|c|c|c|c|}
\hline \multicolumn{3}{|c|}{ Dificuldade } & $\bar{\psi}$ & $\sum^{2}$ & $\mathbb{L}^{+}$ & $\pi$ & $\sum^{\infty}$ & $\stackrel{2}{4}$ & $\frac{m}{4}$ & $\frac{ \pm}{4}$ & $\stackrel{n}{4}$ & $\stackrel{0}{<}$ \\
\hline \multirow{6}{*}{$\begin{array}{l}\text { Desenvolvimento } \\
\text { da forma de } \\
\text { demonstração }\end{array}$} & \multirow{2}{*}{ Argumentação } & Inválida & & & & & & $\mathrm{X}$ & & $\mathrm{X}$ & & $\mathrm{X}$ \\
\hline & & Incompleta & & & $\mathrm{X}$ & $\mathrm{X}$ & & & & & & \\
\hline & \multirow{2}{*}{$\begin{array}{c}\text { Estrutura da } \\
\text { demonstração }\end{array}$} & $\begin{array}{l}\text { Distinção entre } \\
\text { hipótese e tese }\end{array}$ & & & & & & & & & & \\
\hline & & $\begin{array}{l}\text { Distinção entre } \\
\text { definição e } \\
\text { demonstração }\end{array}$ & & & & & & & & & & \\
\hline & \multirow{2}{*}{$\begin{array}{c}\text { Forma de } \\
\text { demonstração }\end{array}$} & $\begin{array}{c}\text { Utiliza } \\
\text { exemplos }\end{array}$ & & & & & & $\mathrm{X}$ & & $\mathrm{X}$ & & $\mathrm{X}$ \\
\hline & & $\begin{array}{c}\text { Forma } \\
\text { inadequada }\end{array}$ & & & & & & & & & & \\
\hline \multirow{3}{*}{$\begin{array}{l}\text { Conhecimento do } \\
\text { conteúdo }\end{array}$} & \multicolumn{2}{|c|}{ Conectivos } & & & & & & & & & & \\
\hline & \multicolumn{2}{|c|}{ Condicional e bicondicional } & & & & & & $\mathrm{X}$ & & & & \\
\hline & \multicolumn{2}{|c|}{ União, interseção, complementar } & & & & & & $\mathrm{X}$ & & $\mathrm{X}$ & & $\mathrm{X}$ \\
\hline \multirow{3}{*}{ Escrita } & \multirow{2}{*}{$\begin{array}{l}\text { Linguagem } \\
\text { materna }\end{array}$} & Clareza & & & & & & & & & & \\
\hline & & Coerência & & & & & & & & & & \\
\hline & \multicolumn{2}{|c|}{ Linguagem matemática } & & & & & & $\mathrm{X}$ & & & & \\
\hline
\end{tabular}

Fonte: Autoria própria

\section{Questão quatro}

4) Seja $f: X \rightarrow Y$ uma aplicação. Demonstre que: $f$ é injetiva se, e somente se, quaisquer que sejam $A, B \subset X, f(A \cap B)=f(A) \cap f(B)$.

Analisando os tipos de resoluções obtidas e a coerência da questão quatro, obtivemos o quadro sete.

Quadro 7 - agrupamentos da questão quatro da $\mathbf{P}_{2}$

\begin{tabular}{|c|c|c|c|c|c|c|c|c|c|c|c|}
\hline \multicolumn{2}{|c|}{ Agrupamentos } & $\overline{4}$ & $\mathbb{4}^{2}$ & \& & 文 & $\gtrless^{\infty}$ & $\stackrel{0}{2}$ & $\frac{m}{4}$ & $\frac{ \pm}{4}$ & $\stackrel{n}{4}$ & $\stackrel{0}{<}$ \\
\hline \multirow{3}{*}{ Coerente } & $C_{1}$ & & & & & & & & & & \\
\hline & $C_{2}$ & & & & & & & & & & \\
\hline & $C_{3}$ & $\mathrm{X}$ & & $\mathrm{X}$ & & & & $\mathrm{X}$ & & $\mathrm{X}$ & \\
\hline \multicolumn{2}{|c|}{ Incoerente } & & $\mathrm{X}$ & & $\mathrm{X}$ & $\mathrm{X}$ & & & $\mathrm{X}$ & & \\
\hline \multicolumn{2}{|c|}{ Em branco } & & & & & & & & & & \\
\hline \multicolumn{2}{|c|}{ Justificada } & & & & & & $\mathrm{X}$ & & & & $\mathrm{X}$ \\
\hline
\end{tabular}

Fonte: Autoria própria 
$\mathrm{Em} \mathrm{C}_{3}$, todos os graduandos apresentaram uma demonstração incompleta, provando apenas uma das condicionais da proposição. Foi possível perceber que na análise da sentença, os sujeitos não diferenciaram hipótese e tese. De modo que, não consideraram que uma bicondicional é formada por duas condicionais.

De acordo com Dreyfus (1991), existe uma dificuldade em gerenciar a complexidade no desenvolvimento de uma prova, assim sendo não atingiram a finalidade de provar a sentença. Para Balacheff (1987), esse seria um exemplo de exemplo genérico, devido à complexidade da questão. Notamos que o registro escrito de $\mathrm{A}_{1}$ apresentou dificuldade notacional; o estudante identifica os elementos pertencentes ao domínio e à imagem da mesma forma, comprometendo a coerência da argumentação.

Já no registro escrito de $\mathrm{A}_{4}$ temos um argumento inválido acerca da definição de interseção, comprometendo a demonstração. Essa questão da segunda proposta de tarefas foi selecionada com a intenção de analisarmos como os estudantes gerenciariam a complexidade de uma proposição. Havia a necessidade de provar duas condicionais, sendo que em uma dessas condicionais existiam duas inclusões de conjuntos a serem demonstradas. Nenhum dos participantes demonstrou todas as partes da proposição; obtivemos apenas provas parciais, evidenciando dificuldade em lidar com um problema complexo a resolver.

Notemos o registro escrito a seguir que apresentou a demonstração para uma das condicionais da proposição.

Figura 5- Registro escrito de $A_{13}$

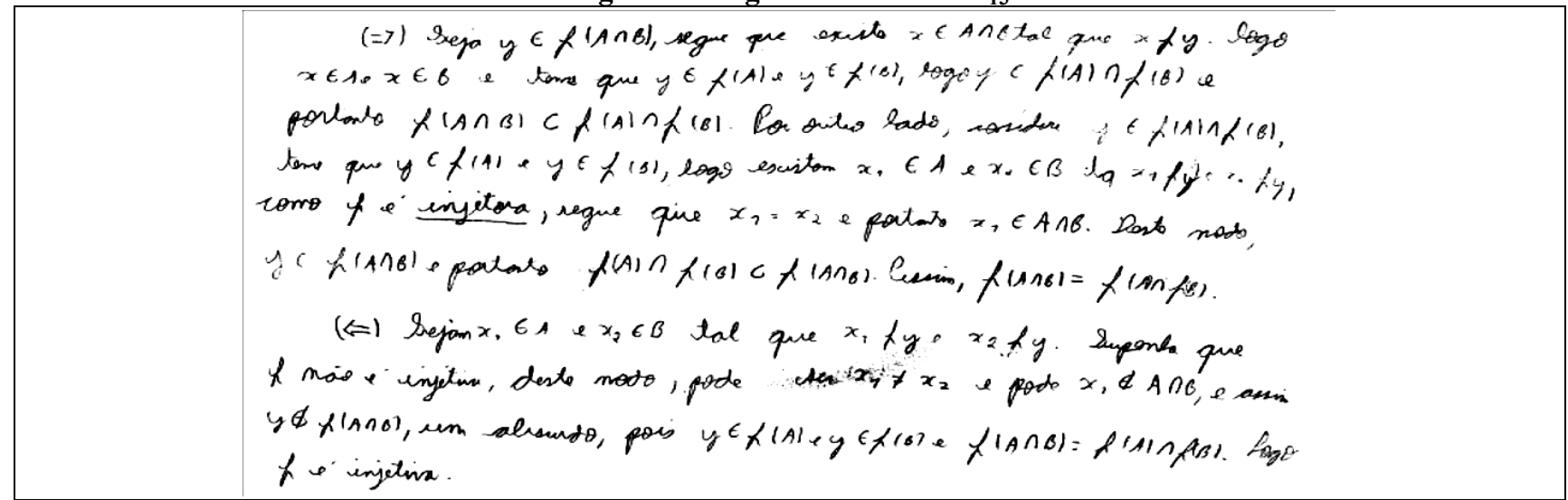

Fonte: registro escrito de $A_{13}$ : questão quatro da $P_{2}$

Os demais integrantes da investigação apresentaram respostas incoerentes, ou seja, não argumentaram adequadamente ou apenas justificaram a proposição. No quadro oito temos uma síntese das dificuldades evidenciadas nessa questão. 
Quadro 8 - dificuldades encontradas na questão quatro da $\mathbf{P}_{2}$

\begin{tabular}{|c|c|c|c|c|c|c|c|c|c|c|c|c|}
\hline \multicolumn{3}{|l|}{ Dificuldade } & $\overline{4}$ & $\sum^{2}$ & $\mathbb{4}^{+}$ & 交 & $i^{\infty}$ & $\frac{0}{4}$ & $\sum^{m}$ & $\stackrel{\Delta}{\Delta}$ & $\frac{n}{k}$ & $\stackrel{0}{\mathbb{4}}$ \\
\hline \multirow{6}{*}{$\begin{array}{l}\text { Desenvolvimento da } \\
\text { forma } \\
\text { demonstração }\end{array}$} & \multirow[t]{2}{*}{ Argumentação } & Inválida & & & & & & & & & & \\
\hline & & Incompleta & $\mathrm{X}$ & & $\mathrm{X}$ & & & & $\mathrm{X}$ & & $\mathrm{X}$ & \\
\hline & \multirow[t]{2}{*}{$\begin{array}{l}\text { Estrutura da } \\
\text { demonstração }\end{array}$} & $\begin{array}{l}\text { Distinção entre } \\
\text { hipótese e tese }\end{array}$ & $\mathrm{X}$ & & $\mathrm{X}$ & & & & $\mathrm{X}$ & & $\mathrm{X}$ & \\
\hline & & $\begin{array}{l}\text { Distinção entre } \\
\text { definição e } \\
\text { demonstração }\end{array}$ & & & & & & & & & & \\
\hline & \multirow[t]{2}{*}{$\begin{array}{l}\text { Forma de } \\
\text { demonstração }\end{array}$} & $\begin{array}{l}\text { Utiliza } \\
\text { exemplos }\end{array}$ & & & & & & & & & & \\
\hline & & $\begin{array}{l}\text { Forma } \\
\text { inadequada }\end{array}$ & $\mathrm{X}$ & & $\mathrm{X}$ & & & & & & & \\
\hline \multirow{3}{*}{$\begin{array}{l}\text { Conhecimento do } \\
\text { conteúdo }\end{array}$} & \multicolumn{2}{|l|}{ Conectivos } & & & & & & & & & & \\
\hline & \multicolumn{2}{|c|}{ Condicional e bicondicional } & $\mathrm{X}$ & & $\mathrm{X}$ & & & & $\mathrm{X}$ & & $\mathrm{X}$ & \\
\hline & \multicolumn{2}{|c|}{ União, interseção, complementar } & & & $\mathrm{X}$ & & & & & & & \\
\hline \multirow[t]{3}{*}{ Escrita } & \multirow{2}{*}{$\begin{array}{l}\text { Linguagem } \\
\text { materna }\end{array}$} & Clareza & & & & & & & & & & \\
\hline & & Coerência & $\mathrm{X}$ & & & & & & & & & \\
\hline & \multicolumn{2}{|c|}{ Linguagem matemática } & $X$ & & & & & & & & & \\
\hline
\end{tabular}

Fonte: Autoria própria

\section{Considerações finais}

Tínhamos como objetivo detectar dificuldades encontradas por graduandos em tarefas envolvendo demonstrações matemáticas. Para atingir esse objetivo, categorizamos e sintetizamos os dados obtidos nos registros escritos dos graduandos e detectamos dificuldades relacionadas: à forma de demonstração, ao conteúdo e à escrita na linguagem matemática ou materna, explicitadas nas análises, o que fica evidente a partir do quadro quatro.

Quanto à frequência dessas dificuldades, a argumentação foi o item mais frequente, aparecendo em vinte e quatro registros escritos, seguido do item bicondicional e condicional e do item união, interseção e complementar, que apareceram catorze vezes cada. Ao todo tivemos duzentos e dezenove registros escritos considerando treze participantes da P1, que continha treze questões e dez participantes da P2 com cinco questões. Cabe ressaltar que na P1, onze das treze questões não contemplavam demonstrações, apenas abordavam uma parte inicial do conteúdo tratado, qual seja, conjuntos e funções. Assim, ficamos com setenta e seis registros escritos contemplando demonstrações. Os itens que apresentaram menos dificuldades foram clareza e coerência na linguagem materna.

Detectamos resoluções de questões que não apresentavam tais dificuldades, nestes casos, analisamos o desenvolvimento da demonstração e focamos no P.M.A. envolvido em cada 
proposição. Os processos de representação apareceram com mais frequência do que os processos de abstração.

Explicitando dificuldades em demonstrações matemáticas que podem ser comuns a inúmeros outros graduandos de cursos de Matemática, a investigação relatada neste artigo espera contribuir com a comunidade acadêmica, com o intuito de instigar a reflexão sobre o processo de ensino e aprendizagem acerca das demonstrações.

\section{Referências}

BALACHEFF, N./ Processus de preuve et situations de validation. In: Educational Studies in Mathematics, v. 18, n. 2, p.147-176, 1987.

BALACHEFF, N. / The researcher epistemology: a deadlock for educational research on proof. Les Cahiers du Laboratoire Leibniz, Grenoble, n. 109, 2004.

DOMInGUES, H. H. ; IEZZI, G./ Álgebra Moderna. 4a ed. São Paulo: Atual, 2003.

DREYFUS, T./ Advanced Mathematical Thinking Processes. In: Tall, D./ Advanced mathematical thinking. Dordrecht: Kluwer, p. 25-41, 1991.

HANNA, G., BARBEAU, E./ Proofs as bearers of mathematical knowledge. ZDM Mathematics Education, n. 40, p. $345-353,2008$.

MARIOTTI, M. A.; BALACHEFF, N./ Introduction to the special issue on didactical and epistemological perspectives on mathematical proof. ZDM Mathematics Education, Heidelberg, v.40, n. 3, p. 341-344, 2008.

MOREIRA, P. C.; DAVID, M. M. M.S./ A formação matemática do professor: licenciatura e prática docente escolar. Belo Horizonte: Autêntica, p. 22-23, 2005.

PONTE, J. P.; BOAVIDA, A.; GRAÇA, M. \& ABRANTES, P./ Didática da Matemática. Lisboa: DES do ME., 1997.

RAV, Y./ Why Do We Prove Theorems? Philosophia Mathematica, Oxford, 1999,

TALL, D./ The psychology of advanced mathematical thinking. In: Tall, D./ Advanced mathematical thinking. Dordrecht: Kluwer, p. 3-21, 1999.

TALL, D/ Cognitive Growth in Elementary and Advanced Mathematical Thinking. Plenary

Lecture, Conference of the International Group for the Psychology of Learning Mathematics, Recife, Brazil, v. 1, p. 161-175, 1995.

Submetido em novembro de 2014

Aprovado em dezembro de 2014 